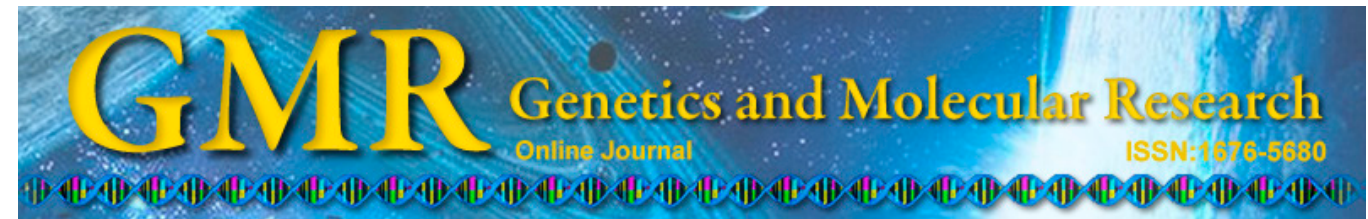

\title{
Chromosome mapping of 18S rDNA and 5S rDNA by dual-color fluorescence in situ hybridization in the half-smooth tongue sole (Cynoglossus semilaevis)
}

\author{
L. Jiang*, J. Jiang*, J. Liu, J. Yuan, Y. Chen, Q. Zhang and X. Wang
}

Key Laboratory of Marine Genetics and Breeding, Ocean University of China, Ministry of Education, Qingdao, China

*These authors contributed equally to this study.

Corresponding author: X. Wang

E-mail: wangxubo@ouc.edu.cn

Genet. Mol. Res. 13 (4): 10761-10768 (2014)

Received November 21, 2013

Accepted May 27, 2014

Published December 18, 2014

DOI http://dx.doi.org/10.4238/2014.December.18.17

\begin{abstract}
Half-smooth tongue sole (Cynoglossus semilaevis) is an important aquaculture flatfish in China. Cytogenetic analysis has revealed that its sex determination system is female heterogametic (ZZ/ZW). The $\mathrm{W}$ chromosome is morphologically larger and has been considered evolutionarily younger than any other chromosome in the set. However, the genetic origin and evolution process of this neo-chromosome remains unclear. In this study, 2 tandem arrays of rRNA genes were chosen to address this question. Both the major rDNA (18S rDNA) and the minor rDNA (5S rDNA) were located on the C. semilaevis chromosomes by fluorescence in situ hybridization (FISH). Six 18S rDNA signals were observed on the centromeric regions of 3 pairs of autosomes in both males and females. In females, there was an additional $18 \mathrm{~S}$ rDNA signal mapping to the telomeric region of the $\mathrm{W}$ chromosome long arm. With respect to the $5 \mathrm{~S}$ rDNA, 12 signals were mapped to the centromeric regions of six pairs of autosomes. Two-color FISH further confirmed that the two pairs
\end{abstract}


of the $5 \mathrm{~S} \mathrm{rDNA}$ signals were correspondingly located at the same positions of the same autosomes as those of the $18 \mathrm{~S}$ rDNA signals. These results allowed us to speculate about the evolution process of the $\mathrm{W}$ chromosome. Chromosome fusions and repetitive sequence accumulations might have occurred in C. semilaevis. The synteny and non-synteny of $C$. semilaevis $18 \mathrm{~S}$ rDNA and 5S rDNA might imply the original and evolutionary characteristics of this species. These findings will facilitate studies on karyotype evolution of the order Pleuronectiformes.

Key words: Fluorescence in situ hybridization; Major rDNA; Minor rDNA; Pleuronectiformes; Sex chromosome; Cynoglossus semilaevis

\section{INTRODUCTION}

Half-smooth tongue sole (Cynoglossus semilaevis Günther, 1873), a member of Pleuronectiformes, Soleoidei, Cynoglossidae, Cynoglossus, is one of the most commercially important aquaculture fish species in China. As a hotspot of molecular research, it has been studied from many aspects, such as the development of molecular DNA markers (Zhong et al., 2009), construction of fosmid libraries (Wang et al., 2009a), and creation of genetic linkage map (Liao et al., 2009). However, because of the existence of many small and similar chromosomes (Pardo et al., 2001), cytogenetic investigations of this species are impeded by the difficulties of karyotype analysis.

Chromosomal observations revealed that the karyotype of $C$. semilaevis was $2 n=42$, $\mathrm{NF}=42$ (Zhou et al., 2005). Cytogenetic and genetic analyses revealed that its sex determination system is female heterogametic (ZZ/ZW) (Zhuang et al., 2006), with females possessing a $\mathrm{W}$ chromosome that is obviously larger than all the other chromosomes. Although some of the other genetically and/or cytogenetically studied Pleuronectiformes species possess obvious XX/ $\mathrm{XY}$ or ZZ/ZW genetic sex determination systems, it was noteworthy that no distinct dimorphic sex chromosome has been identified (Kikuno et al., 1986; Bouza et al., 1994; Yamamoto, 1999; Carvalho et al., 2005). Therefore, the distinct heteromorphic W chromosome in C. semilaevis is evolutionarily a neo-sex chromosome, which might evolve during or after speciation. Although several sex-specific probes have been developed from the W chromosome (Wang et al., 2009b; Zhai et al., 2011) of C. semilaevis, the origin and evolution of this neo-chromosome remains unknown. To uncover the genetic basis of this chromosome, as well as the evolutionary status and taxonomy position of this species, further cytogenetic studies must be conducted.

Tandem arrays of rRNA genes are organized into 2 distinct multi-gene families composed of hundreds to thousands of copies. One family is represented by the major rDNA, $45 \mathrm{~S}$ rDNA, which encodes the $18 \mathrm{~S}, 5.8 \mathrm{~S}$, and $28 \mathrm{~S}$ rRNAs. The second family is represented by the minor rDNA, 5S rDNA, which encodes the 5S rRNA (Martins and Galetti, 2001). There are usually one or more pairs of rDNA sites in the genome, which can be easily located by fluorescence in situ hybridization (FISH) with labeled DNA probes. The 5S rDNA repeats comprise conserved coding sequences of 120 base pairs (bp), which are separated from each other by non-transcribed spacer (NTS) sequences (Inafuku et al., 2000). It has been demonstrated that these conserved sequences can also be located by the NTS (Suzuki et al., 1996). The rRNA genes are worth studying because the sequences (particularly in the intergenic spacer region and NTS 
region), the copy number of the repeats at each site, and the number of sites can evolve rapidly. Detecting the variation will help to explain some karyotype rearrangement processes that may be difficult to identify with other genetic marker methods (Galasso et al., 1995).

This study aimed to locate the $18 \mathrm{~S}$ and $5 \mathrm{~S}$ rDNAs on the chromosomes of C. semilaevis and to speculate about the chromosome evolution of this species.

\section{MATERIAL AND METHODS}

\section{Sampling and chromosome preparation}

Ten wild individuals ( 5 females and 5 males) of C. semilaevis were collected from the Yellow Sea adjacent to Yantai City, China. Their weights ranged from 350 to $600 \mathrm{~g}$, and their body length ranged from 40 to $60 \mathrm{~cm}$. Chromosome preparations were performed following the method of Wang et al. (2009c).

\section{Probe labeling}

The 18S rDNA and NTS primers (18S-F: 5'-GAGAAACGGCTACCACATC-3', 18S-R: 5'-ACAAATCGCTCCACCAAC-3', NTS-F: 5'-GGAGACCGCCTGGGAATAC-3', and NTS-R: 5'-ATCGGGCGTGTTCAGGGTG-3') were designed according to the homologous sequences of Japanese flounder (Accession No. EF126037 and AB154836). The $20 \mu \mathrm{L}$ polymerase chain reaction (PCR) mixture contained $40 \mathrm{ng}$ genomic DNA, $0.2 \mathrm{mM}$ of each primer, $200 \mu \mathrm{M}$ dNTPs, 2.5 $\mathrm{mM} \mathrm{Mg}^{2+}$, 1X PCR Buffer, and $1 \mathrm{U}$ rTaq (TaKaRa, Dalian, China). The PCR conditions were as follows: 1 cycle of denaturation at $94^{\circ} \mathrm{C}$ for $5 \mathrm{~min} ; 30$ cycles of $30 \mathrm{~s}$ at $94^{\circ} \mathrm{C}, 30 \mathrm{~s}$ at $56^{\circ} \mathrm{C}$, and 30 s at $72^{\circ} \mathrm{C}$, with a final extension step at $72^{\circ} \mathrm{C}$ for $15 \mathrm{~min}$. Products of about 900 and $800 \mathrm{bp}$ could be separately amplified from the $C$. semilaevis genome. Via cloning, sequencing, and basic local alignment search tool analysis in the National Center for Biotechnology Information database, the 2 PCR products were demonstrated to be $18 \mathrm{~S}$ rDNA and NTS sequences, respectively. The verified products were purified with the Cycle-Pure Kit (Omega, GA, USA). Probes for 18S rDNA and NTS were prepared with purified PCR products by DIG-Nick Translation Mix or Biotin-Nick Translation Mix according to manufacturer protocols (Roche, Mannheim, Germany). The labeled probes were precipitated with isopropyl alcohol and ammonium acetate at $-20^{\circ} \mathrm{C}$ for $12 \mathrm{~h}$ and dissolved with $50 \%$ dextran sulfate and $20 \mathrm{X}$ saline sodium citrate $(\mathrm{SSC})(\mathrm{v} / \mathrm{v})$ at $4^{\circ} \mathrm{C}$.

\section{Fluorescence in situ hybridization (FISH)}

FISH and probe detection were conducted according to Wang et al. (2009c) and $\mathrm{Hu}$ et al. (2011) with minor modifications. Aged slides were treated with $0.005 \%$ pepsin in 10 $\mathrm{mM} \mathrm{HCl}$ at $37^{\circ} \mathrm{C}$ for $10 \mathrm{~min}$. Then, they were washed 2 times with phosphate-buffered saline, rinsed in 70 and $100 \%$ ethanol each for $5 \mathrm{~min}$, and air-dried. RNase-treated slides were denatured in $2 \mathrm{X} \mathrm{SSC}$ containing $70 \%$ formamide at $75^{\circ} \mathrm{C}$ for 2 to $3 \mathrm{~min}$, dehydrated with a precooled ethanol series of concentration gradients $(70,90$, and $100 \%)$ for $5 \mathrm{~min}$ each, and air-dried. The $20 \mu \mathrm{L}$ probe hybridization mix (containing about $10 \mathrm{ng} / \mu \mathrm{L}$ probes, $10 \%$ dextran sulfate, and $50 \%$ deionized formamide in $2 \mathrm{X} \mathrm{SSC}$ ) was denatured at $95^{\circ} \mathrm{C}$ for $5 \mathrm{~min}$ and chilled on ice immediately for at least $10 \mathrm{~min}$. The denatured probe was applied to the slide under a cover slip. DNA-DNA in situ hybridization was performed in a humidity chamber at 
$37^{\circ} \mathrm{C}$ for 12 to $16 \mathrm{~h}$. After hybridization, the slides were washed in $2 \mathrm{X}$ SSC with $50 \%$ formamide at $37^{\circ} \mathrm{C}$ for $10 \mathrm{~min}, 1 \mathrm{X} \mathrm{SSC}$ at $37^{\circ} \mathrm{C} 3$ times for $5 \mathrm{~min}$ each, and $2 \mathrm{X} \mathrm{SSC}$ at room temperature for 1 to $2 \mathrm{~min}$. The hybridized probes that were labeled with biotin or digoxigenin were detected with fluorescein isothiocyanate (Roche) or antidigoxigenin rhodamine (Roche) in a humidity chamber at $37^{\circ} \mathrm{C}$ for $1 \mathrm{~h}$. Then, the slides were washed in washing buffer $(0.1 \%$ Tween 20 in $4 \mathrm{X} \mathrm{SSC})$ at $37^{\circ} \mathrm{C} 3$ times for 5 min each and $2 \mathrm{X} \mathrm{SSC}$ for 1 to $2 \mathrm{~min}$ at room temperature. Chromosomes were counterstained with propidium iodide (only biotin) or 4',6-diamidino-2-phenylindole (both biotin and digoxigenin) for $10 \mathrm{~min}$ at room temperature in a cassette. Slides were observed using a Nikon E-600 epifluorescence microscope equipped with a $\mathrm{CCD}$ camera (COHU). The signals were collected using appropriate filter sets and the LUCIA software. At least 20 metaphases were examined in each individual.

\section{RESULTS}

The karyotype of $C$. semilaevis was observed to be $2 \mathrm{n}=42$ acrocentric chromosomes, which was in accordance with previous results (Zhou et al., 2005; Wang et al., 2009b). Compared to males, females had a unique and larger $\mathrm{W}$ chromosome with no homologous chromosome in the genome (Figure 1A). FISH with the 18S rDNA probe showed signals in the centromeric or subcentromeric regions of 3 pairs of autosomes in males (Figure 1B). However, in females, 7 signals were found in each metaphase spread, including 6 in the centromeric regions of 3 autosomal pairs and another signal in the subtelomeric region of the $\mathrm{W}$ chromosome long arm (Figure 1A). The 5S rDNA loci were detected on more chromosomes than the $18 \mathrm{~S}$ rDNA loci. In total, 12 signals were identified in both the male and female genome, all of which were located in the centromeric region of autosomes (Figure 1C). No hybridization signal was found on the W chromosome. The signal intensities or "sizes" varied greatly among different chromosomes of the same metaphase spread, but the FISH results were consistent both among metaphases and among the 10 specimens. No individual-specific hybridization signal was observed.

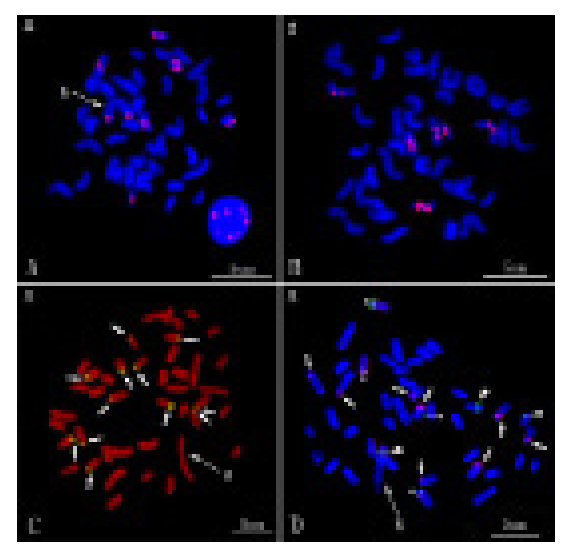

Figure 1. Distribution of $18 \mathrm{~S} \mathrm{rDNA}$ and $5 \mathrm{~S} \mathrm{rDNA}$ by fluorescence in situ hybridization (FISH) in mitotic chromosomes of Cynoglossus semilaevis. (A) 18S rDNA signals on the female metaphase spread, (B) 18S rDNA singnals on the male metaphase spread, (C) 5S rDNA signals on the female metaphase spread, and (D) 18S rDNA and 5S rDNA dual-color FISH signals on a female metaphase spread. The long arrows indicate $\mathrm{W}$ chromosomes, short arrows indicate 5S rDNA signals, arrow heads indicate $18 \mathrm{~S}$ rDNA signals, and asterisks indicate $18 \mathrm{~S}$ rDNA and $5 \mathrm{~S}$ rDNA sympatric signals located at the same position. $\mathrm{Bar}=5 \mu \mathrm{m}$. 
Two-color FISH with 18S rDNA and 5S rDNA probes revealed that only the $18 \mathrm{~S}$ rDNA signal could be identified on the $\mathrm{W}$ chromosome in the female genome. Syntenic signals of the 2 probes could be found on 2 pairs of autosomes. Besides, signals of $18 \mathrm{~S}$ rDNA were also located on another pair of autosomes, and those of 5S rDNA were observed on another 4 pairs of autosomes (Figure 1D).

\section{DISCUSSION}

According to the existing cytogenetic studies in the order Pleuronectiformes, the $2 \mathrm{n}$ $=48$ telocentric karyotype has been proposed to be ancestral (Pardo et al., 2001). The diploid number is relatively conserved in families Pleuronectidae and Paralichthyidae, ranging from $2 \mathrm{n}=44$ to $2 \mathrm{n}=48$. However, the diploid number is rather variable in Cynoglossidae, Scophthalmidae, Soleidae, and Bothidae, ranging from $2 n=28$ to $2 n=48$ (Pardo et al., 2001). In Scophthalmus maximus $(2 \mathrm{n}=44), 2$ centric fusions have been proposed to explain the evolution of the turbot karyotype (Bouza et al., 1994). In Achiridae $(2 n=34-40)$ and Soleidae $(2 n=42)$, the reduction in the diploid number is possibly due to chromosome fusions in the ancestor of these families (Pardo et al., 2001; Carvalho et al., 2005). Therefore, it is rational to speculate that chromosome fusions may also occur in C. semilaevis. It has also been reported that sex chromosomes are labile and that their formation in fish is often associated with the accumulation of repetitive sequences or long interspersed repeat elements (Carvalho et al., 2005; Ellegren, 2011; Takehana et al., 2012) and the silencing of genes (Zhang et al., 2001). This study of the $C$. semilaevis $\mathrm{W}$ chromosome showed that a number of repetitive sequences accumulated in heterochromatic regions (data not shown), which was also found in mammals, birds, and other fishes (Mank et al., 2006; Ellegren, 2011).

In aquatic animals, especially in fishes, the numbers and locations of rDNA loci on chromosomes vary significantly. In Paralichthyidae (Wang et al., 2009c), Soleidae, and Scophthalmidae (Pardo et al., 2001), the 18S rDNA is generally located on 1 pair of autosomes. In Apareiodon ibitiensis, populations from different areas have 1 or 2 pairs of $18 \mathrm{~S}$ rDNA FISH signals (Bellafronte et al., 2009). This may be caused by chromosome rearrangements and consequent dispersion throughout the genome (Moreira-Filho et al., 1984). As reported in many species, chromosome evolution from geographical isolation or reproductive isolation generates multiple 18S rDNA sites (Sola et al., 2000; Vicari et al., 2005). Three pairs of $18 \mathrm{~S}$ rDNA signals and 6 pairs of 5S rDNA signals found in this study implied that the C. semilaevis genome had experienced a series of chromosome rearrangements and consequent dispersion. This phenomenon might result from chromosome translocation, insertion, or some undiscovered events during evolution among homologous or non-homologous chromosomes. It was interesting that the telomeric regions of the $\mathrm{W}$ chromosome long arm showed an explicit signal in all analyzed metaphases of females. The same phenomenon has been reported in Oryzias hubbsi and O. javanicus (Takehana et al., 2012), with 3 18S rDNA sites located on a pair of autosomes and the $\mathrm{W}$ chromosome in females. In Triportheus auritus, $518 \mathrm{~S}$ rDNA fluorescence hybridization signals are present on 2 autosomal pairs and the $\mathrm{W}$ chromosome (Cioffi et al., 2012). A sex chromosome bearing rDNA loci has also been found in other fish species, such as Fundulus diaphanous (Howell and Black, 1979) and Hoplias malabaricus (Born and Bertollo, 2000).

The existence of $18 \mathrm{~S}$ rDNA on the "evolutionarily young" W chromosome may result from chromosome translocation between non-homologous chromosomes after a novel W chro- 
mosome is formed. Then, the $18 \mathrm{~S}$ rDNA amplifies on the neo-W chromosome. The author of an existing study inclines to the hypothesis that nucleolus-organizing regions associated with terminal heterochromatin were more prone to chromosome rearrangements (Moreira-Filho et al., 1984). Besides, the accumulations of repetitive sequences on independently evolved sex chromosomes have been proved (Cioffi et al., 2012). The chromosome fusions and repetitive sequences accumulations may explain, to some extent, why the $\mathrm{W}$ chromosome was larger than the $\mathrm{Z}$ chromosome and autosomes.

The $5 \mathrm{~S}$ rDNA locus has been considered to be located on 1 pair of chromosomes in most organisms including fishes. Martins and Galetti (1999) regard this as an ancient phenomenon in fishes. In the family Curimatidae, Steindachnerina insculpta has 1 pair of 5S rDNA sites and Cyphocharax modesta has 2 pairs (Santos et al., 2006). In the order Tetraodontiformes, Sphoeroides greeleyi and S. spinosus possess 1 pair of 5S rDNA sites, while Cyclichthys spinosus possesses 2 pairs (Noleto et al., 2007). In the order Pleuronectiformes, Paralichthys olivaceus (Paralichthyidae) and Solea solea (Soleidae) have 1 pair of 5S rDNA sites (Libertini et al., 2002; Fujiwara et al., 2007). Presumably, the 6 pairs of 5S rDNA signals in C. semilaevis might also have been generated from chromosome rearrangement, like the 18S rDNA. These 5S rDNA signals were all located in interstitial autosome segments, as observed in other fishes (Fujiwara et al., 1998; Martins and Galetti, 2001). Such a pattern may protect 5S rDNA clusters from evolutionary events (Noleto et al., 2007). The signal strength of hybridization that was observed in this study was variable in different positions, which might result from different copy numbers of the sequence. A similar situation exists in salmonid fish, where a lower copy number of rDNA clusters may lead to weaker signals (Fujiwara et al., 1998).

It was intriguing to find that 2 pairs of $18 \mathrm{~S}$ rDNA and 2 pairs of $5 \mathrm{~S}$ rDNA sites were correspondingly located at the same position of the $C$. semilaevis chromosomes, while the other signals were detected in different positions. In Salmo salar (Pendas et al., 1994) and Paralichthys olivaceus (Fujiwara et al., 2007), both the major and minor rDNA have 1 pair of signals located on the same chromosomes. In Oncorhynchus mykiss (Fujiwara et al., 1998), there are 2 pairs of 5S rDNA signals, 1 of which is syntenic with $18 \mathrm{~S}$ rDNA and the other is on X chromosomes. In contrast, in Hucho perryi (Fujiwara et al., 1998), Epinephelus marginatus (Sola et al., 2000), Cyclichthys spinosus (Noleto et al., 2007), and many other teleosts, the major and minor rDNA signals are on different chromosome pairs. According to these previous studies, the location patterns between the major and minor rDNA sites are usually diverse among different families and conserved within the same family. In Parodon species, however, the localizations of both rDNAs are species-specific and can serve as genetic markers (Bellafronte et al., 2005). The synteny of $18 \mathrm{~S}$ and 5S rDNA has been considered as an ancestral status in the fish genome (Fontana et al., 2003), but their non-synteny can prevent undesirable translocations of $5 \mathrm{~S}$ sequences within $45 \mathrm{~S}$ rDNA during evolution (Martins and Galetti, 1999). As observed in this study, C. semilaevis had both the original and the evolutionary characteristics. These $5 \mathrm{~S}$ rDNA sites might acquire distinct structures and functions over a long evolutionary process.

Considering the cytogenetic studies on the Pleuronectiformes, the karyotype of $C$. semilaevis, with multiple major and minor rDNA sites and terminal 18S rDNA loci on the long arm of the $\mathrm{W}$ chromosome, might involve manifold rearrangements including translocation, inversion, and fusion. The existing studies on the order Pleuronectiformes were mainly focused on the families Bathidae, Paralichthydae, Soleidae, Achiridae, and Pleuronectidae (Berendzen et al., 2002). There was a lack of rDNA information about the family Cynoglos- 
sidae. Therefore, it remains unclear whether these findings of $18 \mathrm{~S}$ and $5 \mathrm{~S}$ rDNA location in $C$. semilaevis represent the general characteristics of the family Cynoglossidae. Much information is needed to elucidate the evolutionary relationship between $C$. semilaevis and the other flatfish. Intensive studies are necessary to verify how $18 \mathrm{~S}$ rDNA and the copy number of repetitive rDNAs impact the differentiation of the $\mathrm{W}$ chromosome.

\section{ACKNOWLEDGMENTS}

The authors wish to thank Mr. Zhai Jieming of Laizhou Mingbo Aquatic Co. LTD. for generously providing the specimens that were used. Research supported by the National Natural Science Foundation of China (\#30901098 and \#31272646) and the '863' Hi-Tech Research and Development Program of China (\#2012AA10A402).

\section{REFERENCES}

Bellafronte E, Margarido VP and Moreira-Filho O (2005). Cytotaxonomy of Parodon nasus and Parodon tortuosus (Pisces, Characiformes). A case of synonymy confirmed by cytogenetic analyses. Genet. Mol. Biol. 28: 710-716.

Bellafronte E, Vicari MR, Artoni RF, Margarido VP, et al. (2009). Differentiated ZZ/ZW sex chromosomes in Apareiodon ibitiensis (Teleostei, Parodontidae): cytotaxonomy and biogeography. J. Fish Biol. 75: 2313-2325.

Berendzen PB, Dimmick WW and McEachran JD (2002). Phylogenetic relationships of Pleuronectiformes based on molecular evidence. Copeia 3: 642-652.

Born GG and Bertollo LAC (2000). An XX/XY sex chromosome system in a fish species, Hoplias malabaricus, with a polymorphic NOR-bearing X chromosome. Chromosome Res. 8: 111-118.

Bouza C, Sanchez L and Martínez P (1994). Karyotypic characterization of turbot (Scophthalmus maximus) with conventional, fluorochrome and restriction endonuclease-banding techniques. Mar. Biol. 120: 609-613.

Carvalho AM, Oliveira C, Pardo BG, Martínez P, et al. (2005). Chromosome banding and 18S rDNA in situ hybridization analysis of seven species of the family Achiridae (Teleostei: Pleuronectiformes). Genetica 125: 125-132.

Cioffi MB, Kejnovský E, Marquioni V, Poltronieri J, et al. (2012). The key role of repeated DNAs in sex chromosome evolution in two fish species with ZW sex chromosome system. Mol. Cytogenet. 5: 28.

Ellegren H (2011). Sex-chromosome evolution: recent progress and the influence of male and female heterogamety. Nat. Rev. Genet. 12: 157-166.

Fontana F, Lanfredi M, Congiu L, Leis M, et al. (2003). Chromosomal mapping of 18S-28S and 5S rRNA genes by twocolour fluorescent in situ hybridization in six sturgeon species. Genome 46: 473-477.

Fujiwara A, Abe S, Yamaha E, Yamazaki F, et al. (1998). Chromosomal localization and heterochromatin association of ribosomal RNA gene loci and silver-stained nucleolar organizer regions in salmonid fishes. Chromosome Res. 6 : 463-471.

Fujiwara A, Fujiwara M, Nishida-Umehara C, Abe S, et al. (2007). Characterization of Japanese flounder karyotype by chromosome bandings and fluorescence in situ hybridization with DNA markers. Genetica 131: 267-274.

Galasso I, Schmidt T, Pignone D and Heslop-Harrison JS (1995). The molecular cytogenetics of Vigna unguiculata (L.) Walp: the physical organization and characterization of 18s-5.8s-25s rRNA genes, $5 \mathrm{~s}$ rRNA genes, telomere-like sequences, and a family of centromeric repetitive DNA sequences. Theor. Appl. Genet. 91: 928-935.

Howell WM and Black DA (1979). Location of the nucleolus organizer regions on the sex chromosomes of the banded killifish Fundulus diaphanous. Copeia 1979: 544-546.

Hu L, Shang W, Sun Y, Wang S, et al. (2011). Comparative cytogenetics analysis of Chlamys farreri, Patinopecten yessoensis, and Argopecten irradians with Ct-1 DNA by fluorescence in situ hybridization. Evid. Based Complement. Alternat. Med. 2011: 785831.

Inafuku J, Nabeyama M, Kikuma Y, Saitoh J, et al. (2000). Chromosomal location and nucleotide sequences of 5S ribosomal DNA of two cyprinid species (Osteichthyes, Pisces). Chromosome Res. 8: 193-199.

Kikuno T, Ojima Y and Yamashita N (1986). Chromosomes of flounder, Paralichthys olivacceus. P. Jpn. Acad. 62: 194196.

Liao X, Ma H, Xu G, Shao C, et al. (2009). Construction of a genetic linkage map and mapping of a female-specific DNA marker in half-smooth tongue sole (Cynoglossus semilaevis). Mar. Biotechnol. 11: 699-709.

Libertini A, Mandrioli M, Colomba MS, Bertotto D, et al. (2002). A cytogenetic study of the common sole, Solea solea, 
from the Northern Adriatic Sea. Chromosome Sci. 6: 63-66.

Mank JE, Promislow DEL and Avise JC (2006). Evolution of alternative sex-determining mechanisms in teleost fishes. Biol. J. Linn. Soc. 87: 83-93.

Martins C and Galetti PM (1999). Chromosomal localization of 5S rDNA genes in Leporinus fish (Anastomidae, Characiformes). Chromosome Res. 7: 363-367.

Martins C and Galetti PM (2001). Organization of 5S rDNA in species of the fish Leporinus: two different genomic locations are characterized by distinct nontranscribed spacers. Genome 44: 903-910.

Moreira-Filho O, Bertollo LAC and Galetti PM (1984). Structure and variability of nucleolar organizer regions in Parodontidae fish. Can. J. Genet. Cytol. 26: 564-568.

Noleto RB, Vicari MR, Cipriano RR, Artoni RF, et al. (2007). Physical mapping of 5S and 45S rDNA loci in pufferfishes (Tetraodontiformes). Geneitca 130: 133-138.

Pardo BG, Bouza C, Castro J, Martínez P, et al. (2001). Localization of ribosomal genes in Pleuronectiformes using Ag-, CMA3-banding and in situ hybridization. Heredity 86: 531-536.

Pendas AM, Moran P, Freije JP and Garcia-Vazquez E (1994). Chromosomal mapping and nucleotide sequence of two tandem repeats of Atlantic salmon 5S rDNA. Cytogenet. Cell Genet. 67: 31-36.

Santos LVR, Foresti F, Wasko AP, Oliveira C, et al. (2006). Nucleotide sequence, genomic organization and chromosome localization of 5S rDNA in two species of Curimatidae (Teleostei, Characiformes). Genet. Mol. Biol. 29: 251-256.

Sola L, Innocentiis S, Gornung E, Papalia S, et al. (2000). Cytogenetic analysis of Epinephelus marginatus (Pisces: Serranidae), with the chromosome localization of the $18 \mathrm{~S}$ and $5 \mathrm{~S}$ rRNA genes and of the (TTAGGG) $)_{\mathrm{n}}$ telomeric sequence. Mar. Biol. 137: 47-51.

Suzuki H, Sakurai S and Matsuda Y (1996). Rat 5S rDNA spacer sequences and chromosomal assignment of the genes to the extreme terminal region of chromosome 19. Cytogenet. Cell Genet. 72: 1-4.

Takehana Y, Naruse K, Asada Y, Matsuda Y, et al. (2012). Molecular cloning and characterization of the repetitive DNA sequences that comprise the constitutive heterochromatin of the $\mathrm{W}$ chromosomes of medaka fishes. Chromosome Res. 20: 71-81.

Vicari MR, Artoni RF and Bertollo LAC (2005). Comparative cytogenetics of Hoplias malabaricus (Pisces, Erythrinidae): a population analysis in adjacent hydrographic basins. Genet. Mol. Biol. 28: 103-110.

Wang X, Zhang Q, Sun X, Chen Y, et al. (2009a). Fosmid library construction and initial analysis of end sequences in female half-smooth tongue sole (Cynoglossus semilaevis). Mar. Biotechnol. 11: 236-242.

Wang X, Zhang Q, Ren J, Jiang Z, et al. (2009b). The preparation of sex-chromosome-specific painting probes and construction of sex chromosome DNA library in half-smooth tongue sole (Cynoglossus semilaevis). Aquaculture 297: 78-84.

Wang X, Zhang Q, Chen Y, Qi J, et al. (2009c). Cytogenetic characterization of olive flounder Paralichthys olivaceus: DNA content, karyotype, AgNORs and location of major ribosomal genes. Acta Oceanol. Sin. 28: 72-77.

Yamamoto E (1999). Studies on sex-manipulation and production of cloned populations in hirame, Paralichthys olivaceus. Aquaculture 173: 235-246.

Zhai T, Wang X, Wang J, Gao J, et al. (2011). Screening and FISH locating of a female-specific fosmid clone of halfsmooth tongue sole. Periodical of Ocean University of China 41: 57-60.

Zhang Q, Nakayama I, Fujiwara A, Kobayashi T, et al. (2001). Sex identification by male-specific growth hormone pseudogene (GH- $\Psi)$ in Oncorhynchus masou complex and a related hybrid. Genetica 111: 111-118.

Zhong Q, Yu Y, Zhang Q, Wang Z, et al. (2009). Isolation and characterization of twenty novel microsatellite markers for half-smooth tongue sole (Cynoglossus semilaevis). Conserv. Genet. 10: 1617-1620.

Zhou L, Yang A, Liu X, Du W, et al. (2005). The karyotype analysis of Cynoglossus semilaevis in China. Journal of Fisheries of China 29: 417-419.

Zhuang ZM, Wu D, Zhang SC, Pang QX, et al. (2006). G-banding patterns of the chromosomes of tonguefish Cynoglossus semilaevis Günther, 1873. J. Appl. Ichthyol. 22: 437-440. 